\title{
LA PERSPECTIVA DE GÉNERO EN LA FORMACIÓN INICIAL DOCENTE: ESTUDIO DESCRIPTIVO DE LAS PERCEPCIONES DEL ALUMNADO
}

\author{
(STUDENT PERCEPTIONS OF GENDER MAINSTREAMING IN INITIAL \\ TEACHER TRAINING: A DESCRIPTIVE STUDY)
}

Cristina Miralles-Cardona

María Cristina Cardona-Moltó

Esther Chiner

Universidad de Alicante

DOI: $10.5944 / e d u c X X 1.23899$

Cómo referenciar este artículo/How to reference this article:

Miralles-Cardona, C.; Cardona-Moltó, M. C. y Chiner, E. (2020). La perspectiva de género en la formación inicial docente: estudio descriptivo de las percepciones del alumnado. Educación $X X 1,23(2), 231-257$, doi: 10.5944/educXX1.23899

Miralles-Cardona, C.; Cardona-Moltó, M. C. \& Chiner, E. (2020). Student perceptions of gender mainstreaming in initial teacher training: a descriptive study. Educación XX1, 23(2), 231-257 doi: 10.5944/educXX1.23899

\section{RESUMEN}

Introducción: A pesar de los compromisos gubernamentales para promocionar la igualdad, la incorporación de la perspectiva de género (PG) a la formación inicial docente sigue estando ausente de los planes de estudio e ideario de las facultades de educación. Esta escasa atención al principio de igualdad representa una auténtica barrera para el logro de los Objetivos de Desarrollo Sostenible (Naciones Unidas, 2015) y para el desarrollo y promoción de una educación sensible al género. El propósito de este estudio fue explorar el grado de implementación del enfoque de género en la formación inicial de docentes desde la perspectiva del alumnado. Método: Se diseñó y validó la escala de Evaluación Sensible a la Formación en Igualdad de Género (ESFIG) y se administró a 601 docentes 
en formación de grado y máster (72\% mujeres y $28 \%$ varones), edad media 24.31 años, de una universidad pública española. Resultados: Los datos revelaron que (1) la ESFIG es un instrumento válido y adecuado para medir el estado de incorporación de la PG a la formación inicial docente y (2) que los participantes perciben neutralidad e indiferencia institucional a la hora de integrar el enfoque de género en la docencia; sin embargo, aunque estiman la preparación en género muy importante y necesaria para su formación, muestran una percepción baja y poco realista de la conciencia de desigualdades asociadas al género en los procesos de enseñanza-aprendizaje. Se hallaron diferencias estadísticamente significativas en las respuestas de los participantes en función de la titulación y el género. Discusión: Los resultados sustentan la utilidad de la ESFIG para su uso como un indicador del estado de integración de la PG en la docencia universitaria, así como un instrumento apto para identificar áreas de necesidad y evaluar el impacto de futuras acciones e intervenciones.

\section{PALABRAS CLAVE}

Género; igualdad de género; perspectiva de género; formación inicial del profesorado; educación superior; validación de instrumentos.

\section{ABSTRACT}

Introduction: Despite government commitments to promote equality, gender mainstreaming (GM) in initial teacher preparation is still absent from curricula and thinking of the faculties of education. This little attention to the principle of equality represents a real barrier to achieve the Sustainable Development Goals (United Nations, 2015) and to develop and promote a gender-sensitive education. The purpose of this study was to explore the degree of implementation of the gender approach in initial teacher training from the student teacher's perspective. Method: The Sensitive Assessment of Gender Equality Training (SAGE) scale was designed and validated, and then administered to 601 undergraduate and graduate student teachers (72\% women and 28\% male), mean age 24.31 years, from a Spanish public university. Results: Data revealed that (1) the SAGE is a valid and adequate instrument to measure the implementation status of gender mainstreaming in initial teacher education, and (2) that participants perceive neutrality and institutional indifference when implementing the gender approach in teaching; however, although they consider gender preparation necessary and very important for their education, they show a low and unrealistic perception of awareness of gender inequalities associated to the process 
of teaching and learning. Statistically significant differences were found in participant responses depending on degree and gender. Discussion: The results support the utility of the SAGE for its use as an indicator of gender mainstreaming in university teaching as well as a suitable instrument to identify areas of need and assess the impact of future actions and interventions.

\section{KEY WORDS}

Gender; gender equality; gender mainstreaming; preservice teacher training; higher education; instrument validation.

\section{INTRODUCCIÓN}

Las políticas para promover la igualdad de género han recibido considerable atención en las últimas décadas. Desde los setenta, la igualdad ha sido un objetivo prioritario de la política internacional reconocido a partir de una serie de iniciativas lideradas por Naciones Unidas e impulsadas por los movimientos feministas que ayudaron a establecer un nuevo orden de género a nivel mundial, nacional y regional. El punto de partida fue el acuerdo mundial adoptado en 1979, en Nueva York, por la Asamblea General de las Naciones Unidas (Convención sobre la Eliminación de todas las Formas de Discriminación contra la Mujer, CEDAW, Naciones Unidas, 1979), nacido con el propósito de eliminar cualquier tipo de manifestación sexista contra las mujeres. Las estrategias adoptadas para el desarrollo de dichas políticas han ido cambiando con el paso del tiempo, de modo que el énfasis inicial en la discriminación por sexo evolucionó al de las desigualdades de género y el gender mainstreaming o perspectiva de género (PG) emergió como una nueva estrategia para combatir dichas desigualdades y avanzar hacia la igualdad. Desde Pekín 1995 (Cuarta Conferencia Mundial de las Naciones Unidas), la PG ha liderado el comienzo de un renovado esfuerzo por la lucha contra la desigualdad de los géneros, proceso en el que la Unión Europea ha sido pionera. Más recientemente, los marcos revisados de la Educación para Todos y la Agenda 2030 para el Desarrollo Sostenible (UNESCO, 2015a), continuación de la de los Objetivos de Desarrollo del Milenio, siguen dando una posición central a la igualdad de género (Objetivo 5) al reconocerla como un componente esencial de la formación de los futuros docentes.

El ideario de la igualdad de género llegó a España en la década de los ochenta. El Estado español ratificó la CEDAW en 1983 (BOE, 21/03/1984), suscribiendo el compromiso mundial para combatir las desigualdades 
y adoptando el Protocolo Facultativo de la Convención propuesto por la Asamblea General de las Naciones Unidas en octubre de 1999. La integración de España en la Unión Europea en 1986 supuso un gran empuje para la legitimización de las políticas igualitarias y la institucionalización de los organismos de igualdad, de modo que los esfuerzos por hacer cumplir los principios y derechos contemplados en la CEDAW y en la normativa europea culminaron con la publicación por el Estado español de dos leyes orgánicas: la Ley 1/2004 de Medidas de Protección Integral contra la Violencia de Género (BOE, 29/12/2004) y la Ley 3/2007, de 22 de marzo, para la Igualdad Efectiva de Mujeres y Hombres (BOE, 23/03/2007). La Ley 1/2004, en su art. 4, Principios y valores del sistema educativo, Punto 7 , encomienda a las universidades la tarea de formar en igualdad e insta a que en todos los materiales educativos se eliminen los estereotipos sexistas y discriminatorios (art. 6) y a que en los planes de formación inicial y permanente del profesorado se incluya formación específica en igualdad de género (art. 7). El paso decisivo, sin embargo, se dio con la publicación de la Ley Orgánica 3/2007, de Igualdad Efectiva de Hombres y Mujeres que introdujo métodos y estrategias para la incorporación efectiva del enfoque de género en las instituciones: adopción de la perspectiva de género (PG), creación de planes de igualdad, presupuesto para asuntos de género y evaluación de su impacto. Aplicada a la formación universitaria, la PG se refiere a la necesidad de incluir la visión de género tanto en la docencia como en las líneas de actuación de los órganos de gobierno de facultades y departamentos, siendo los planes de igualdad de género los instrumentos comúnmente utilizados para implementarla.

Para comprender mejor este momento histórico Zippel, et al. (2016) distinguen tres períodos en el discurso político de la igualdad. El primero (1980-1995) caracterizado por la identificación de desigualdades y la propuesta de acciones para eliminarlas; el segundo (1996-2005), definido por la adopción de la perspectiva de género (alentada por la celebración de la Cuarta Conferencia Mundial de las Naciones Unidas de 1995 sobre las Mujeres en Pekín) y su concreción y materialización a través de la implementación de planes de igualdad de género; y el tercero (2005 en adelante), marcado por la introducción del concepto de excelencia inclusiva, término que enfatiza la PG no tanto como herramienta política, sino la dimensión práctica y pedagógica de su incorporación a las acciones igualitarias y/o formativas.

El énfasis en la evaluación de impacto de esas políticas llevó a Bustelo (2003) a hablar de tres generaciones de estudios. Uno de los trabajos más completos y significativos de la primera generación fue el llevado a cabo por el Instituto de la Mujer y el grupo de expertos Red2Red Consultores (2004), diseñado con el propósito de proponer un sistema de indicadores válido para medir el sexismo en las escuelas de educación primaria y secundaria y ofrecer una visión conjunta del estado de la siłulaciéndudeiła educación 
para la igualdad en el país. El proyecto permitió detectar la problemática existente en los centros educativos no universitarios e identificar métodos y herramientas para hacer frente al comportamiento sexista, lo que propició que se desarrollaran experiencias piloto y proyectos de coeducación en diversas comunidades autónomas. La segunda generación de estudios viene definida por la evaluación de los planes de igualdad. Se trataba de comprobar si las acciones contempladas en dichos planes se cumplían. Dentro de esta segunda generación, cabe mencionar el estudio de metaevaluación llevado a cabo por Bustelo (2003) que sometió a escrutinio el plan nacional y 10 planes regionales de igualdad o el más reciente de Pastor-Gosálbez, et al. (2020) que analiza la efectividad de los planes de igualdad en la universidad española 10 años después de la publicación de la Ley 3/2007, concluyendo que aunque las universidades han impulsado acciones para fomentar la igualdad, los avances se están viendo limitados por la rigidez y resistencia de las estructuras universitarias. Por último, la tercera generación de estudios aspira a valorar, no solo el cumplimiento de las acciones e iniciativas, sino también sus resultados. En esta generación se incluyen los estudios realizados en la última década en el ámbito de la formación universitaria. Se trata de un conjunto de estudios dispersos que hasta ahora se han orientado a describir las dificultades asociadas a la incorporación de la PG a la docencia universitaria y que ponen de relieve que, aunque la visión de género está cobrando interés, la exploración de su impacto en la educación superior está infra-representada (Lam et al., 2019).

\section{El género como herramienta pedagógica en la educación superior}

Con estas políticas e iniciativas de fondo, los estudios de género han cobrado auge y aparentemente se han convertido en centro de interés de muchas universidades e institutos de investigación. Constituyen un medio para integrar la PG en la docencia universitaria, a la vez que una herramienta para cuestionar y reconstruir significados, a menudo, ocultos o dados por superados en relación al género. El enfoque de género aporta una nueva mirada a la comprensión de la desigualdad al poner de manifiesto que las condiciones desiguales entre hombres y mujeres responden a un sistema de relaciones de poder arraigado en los significados que el orden cultural atribuye a la feminidad y a la masculinidad. La PG ofrece una nueva mirada que enriquece los procesos epistemológicos de creación del conocimiento y otorga nuevas herramientas para el análisis crítico y la comprensión de los problemas originados en la desigualdad. Según Buquet (2011), otorgar al alumnado en formación estas herramientas conceptuales contribuye a mejorar la calidad de su preparación y su competencia para educar con visión de género a las generaciones futuras. 
A pesar del interés pedagógico y social de la integración de la PG en la formación universitaria, en la práctica, el género no ha emergido como una prioridad en los planes de actuación de las universidades, por lo que su incorporación a la academia no ha resultado una tarea fácil (AnguitaMartínez, 2011; Lahelma y Tainio, 2019; Zippel, et al., 2016). El proceso de convergencia con el Espacio Europeo de Educación Superior tampoco resultó ser un móvil suficiente para incorporar la PG en los estudios universitarios, desperdiciándose así la oportunidad que ofrecía el diseño de los nuevos títulos. Investigaciones como la de Garrigues (2010) en la Universidad Jaume I de Castellón, Larrondo y Rivero (2019) en la Universidad del País Vasco, Valdivieso (2016) en la Universidad de Las Palmas, o Verge, et al. (2018) en la Universidad Pompeu Fabra, entre otras, reflejan bien la resistencia a la inclusión del género en el currículum universitario.

La resistencia a la incorporación del enfoque de género en la actividad académica de las universidades puede ser explicada, no solo por la vaguedad y debilidad de las políticas de género para hacer cumplir las normas establecidas en la legislación, sino también por las prácticas arraigadas en las instituciones que todavía protegen el dominio masculino sobre el femenino en las relaciones de poder. Según Lombardo y Mergaert (2013), la resistencia puede tomar forma institucional o individual, explícita o implícita, y adoptar múltiples formas como negación de la necesidad de cambio, rechazo a tomar responsabilidad o simplemente restar importancia a las políticas de igualdad. A nivel individual cabe destacar como principal obstáculo la falta o escasa conciencia de género y de las desigualdades vinculadas al mismo (Silva, et al., 2017), bastante común, tanto en el alumnado como en el profesorado, y que requiere de acciones inmediatas, pues sin percibir los desajustes y desequilibrios de género existentes, es difícil, por no decir imposible, cambiar las ideas sesgadas y estereotipadas que dificultan la visibilidad, empoderamiento y participación en igualdad de las mujeres en la vida universitaria.

\section{Impacto de la perspectiva de género en la docencia universitaria}

En este contexto, resulta fácil comprender la escasa atención y acogida que ha tenido la incorporación de la PG en los estudios universitarios, y la evaluación de su impacto, aunque la producción científica de la investigación en género vaya en aumento (Lam, et al., 2019). En España, los estudios de Larrondo y Rivero (2019) o de Serra, et al. (2018) reflejan bien cuanto decimos. Larrondo y Rivero (2019) analizaron la integración curricular del género en los estudios de periodismo de las universidades públicas y privadas españolas. Los resultados indicaron que solo una cuarta parte de ellas ofrecen en sus planes de estudio una asignatura con enfoque de género 
y que esta generalmente es optativa. De manera similar, la investigación de Serra, et al. (2018), llevada a cabo en el área de la actividad física y el deporte, confirma la exclusión del género de los planes formativos y concluye que dicha exclusión está institucionalizada en la universidad española. La casuística parece ser común a todas las ramas de conocimiento y disciplinas (Atchison, 2013; González-Pérez, 2017; Larrondo y Rivero, 2019; Serra, et al., 2018; Verdonk, et al., 2009) y países de nuestro entorno (BallarínDomingo, 2017; Valdivieso, 2016; Weiner, 2000; Zippel, et al., 2016).

Igualmente, en el campo específico de la formación inicial del profesorado, el discurso de la igualdad y la incorporación del género a la docencia universitaria está prácticamente ausente tanto en los grados de educación infantil y primaria como en los másteres de formación del profesorado de educación secundaria (Gónzález-Pérez, 2017; Gudbjornsdottir, et al., 2017; Kreitz-Sandberg, 2013; Lahelma y Tainio, 2019; Valdivieso, 2016; Weiner, 2000). Sofía Valdivieso y un equipo de colaboradores revisaron en nuestro país la presencia de asignaturas con contenido de género en los planes de estudio de los grados de maestro/a en educación infantil y primaria de 44 universidades españolas. Hallaron que solamente 11 universidades incluían en sus planes asignaturas sobre género/igualdad de género y que, por lo general, eran asignaturas optativas. En otras cinco universidades identificaron asignaturas que, aunque no recogían la palabra género en el título, el enfoque de género tenía cabida en el temario. Otras investigaciones (e.g. Vizcarra, et al., 2015) llegan a conclusiones similares, contribuyendo a reafirmar que el enfoque de género está prácticamente ausente en la formación inicial del profesorado. Los hallazgos van en la línea de los encontrados por Romero y Abril (2008) con anterioridad, quienes constataron que en las facultades de educación el género no tenía cabida en la docencia y que cuando se contemplaba era por iniciativa individual más que por las directrices trazadas en los planes de centro. Dada esta situación, no debe sorprender que el profesorado perciba la inclusión del género en su asignatura como innecesaria o irrelevante (Anguita-Martínez, 2011; González-Pérez, 2017) y que el conocimiento sobre género constituya un tema secundario o periférico diluido en una consideración más genérica de la igualdad.

\section{El presente estudio}

La existencia de múltiples formas de resistencia a la incorporación de la PG a la educación universitaria y, sobre todo, el desconocimiento de en qué medida esta se implementa en la docencia deja claramente en entredicho la capacidad del sistema universitario para formar a docentes capaces de 
educar en igualdad a las generaciones futuras. El presente estudio surgió con el propósito de explorar mediante encuesta el grado de implementación del enfoque de género en el ámbito de la formación del profesorado. Dada la ausencia de instrumentos aptos para la medición del constructo, se optó por diseñar uno ad hoc. Los objetivos que nos planteamos fueron:

1. Elaborar un instrumento válido y fiable para medir el estado de incorporación de la PG en los estudios de formación del profesorado.

2. Analizar y comparar, por titulación y género, las percepciones de las/los estudiantes de grado y máster a través de los siguientes indicadores: (a) sensibilidad institucional a la aplicación de la PG en la docencia, (b) inclusión del género en el plan de estudios, y (c) conciencia de desigualdades de género asociadas a los procesos de enseñanza-aprendizaje.

\section{MÉTODO}

\section{Contexto y participantes}

El estudio se llevó a cabo en la facultad de educación de una universidad pública española en el marco del programa de investigación en docencia universitaria. En dicha facultad, se forma al futuro profesorado que ejercerá la profesión en las etapas de educación infantil, primaria y secundaria. El número de egresados/as por curso de estas titulaciones docentes asciende anualmente a un millar de los cuales el 30\% corresponde a graduados en educación infantil, 30\% en educación primaria y $40 \%$ en educación secundaria. Las Memorias de los títulos verificadas por la ANECA (Agencia Nacional de Evaluación de la Calidad y Acreditación) contemplan en sus objetivos la formación en igualdad, pero en la práctica, el currículum no incorpora el género en sus contenidos formativos, a pesar de ser considerada una competencia transversal relevante para la formación universitaria. El centro oferta una asignatura optativa "Educar en Igualdad de Género" que, por su naturaleza, constituye una elección y no garantiza que todo el alumnado la curse. Aunque la institución cuenta con un Plan de Igualdad, la Facultad de Educación no dispone de plan de género.

Participaron en el estudio un total de 601 estudiantes de los grados de Maestro/a en Educación Infantil ( $n=196)$, Maestro/a en Educación Primaria $(n=202)$ y máster en Formación del Profesorado de Educación Secundaria $(n=203)$ del último año de carrera que representaban el $61 \%$ de las tres cohortes. Su edad oscilaba entre 20 y 54 años $(M=24.31, D T=5.66)$, edad media por sub-muestras de 23.01, 22.49 y 27.40 años (infantil, primaria y 
secundaria, respectivamente). Un $72 \%(n=433)$ eran mujeres y un $28 \%$ $(n=168)$ varones, de nacionalidad española $(96.80 \%)$ y dedicación completa a los estudios (84\%). Todas las menciones elegidas por los participantes (grado y máster) estaban representadas en la muestra. Como grupo, otorgaban mucha importancia a la formación en género ( 9.14 sobre 10), pero solo una minoría compuesta principalmente por alumnado de educación infantil (un tercio aproximadamente) dijo haber cursado la asignatura optativa "Educar en Igualdad de Género" o poseer nociones previas sobre igualdad de género.

\section{Instrumento}

El instrumento utilizado en este estudio fue la escala de Evaluación Sensible a la Formación en Igualdad del Género (ESFIG) diseñada ad hoc. Para su elaboración se adoptó el enfoque de evaluación sensible al género (Martínez y Díaz, 2017) de reciente incorporación a la evaluación de políticas y servicios en el campo de la salud pública. El instrumento fue diseñado para medir percepciones acerca de la implementación de la perspectiva de género en la formación docente. La escala, en su versión final (véase Apéndice 1), consta de 16 ítems agrupados en tres subescalas de cinco, siete y cuatro ítems, respectivamente que miden grado de acuerdo acerca de (a) la sensibilidad institucional a la aplicación de la política de igualdad de género en los centros, (b) la incorporación de la PG en los planes de estudio y (c) la conciencia de desigualdades vinculadas al género en los procesos instructivos.

Desarrollo de la escala. La ESFIG fue diseñada como una medida unidimensional del grado en que los centros de formación del profesorado integran la PG en sus planes formativos. Fue desarrollada siguiendo una secuencia graduada de pasos: (1) revisión de literatura, (2) valoración de expertos y (3) pilotaje. La revisión de la literatura incluyó la identificación de documentos relativos no solo al procedimiento de desarrollo de escalas, sino también a su contenido. Al no identificar en la literatura instrumentos que sirvieran a nuestros propósitos, se decidió elaborar uno nuevo partiendo del sustento de la teoría del Doing Gender (West y Zimmerman, 1987) y de las recomendaciones contenidas en la Guía para la Igualdad de Género en la Formación del Profesorado (UNESCO, 2015b) y en el documento Training for Gender Equality de Naciones Unidas (UN Women Training Center, 2015). Los fundamentos de la teoría del Doing Gender aconsejan analizar el género no solo como un rasgo de la identidad personal, sino también como un sistema social que funciona a tres niveles: socio-cultural (políticas y leyes), relacional (enseñanzas) e individual (conciencia de género), por lo que se decidió que el instrumento debía contener elementos relativos a la política institucional 
sobre género, a la formación con PG, y a la percepción de desigualdades de género en los procesos instructivos. Inicialmente, se redactó un banco de 38 ítems escritos de forma positiva, independiente y unívoca. Para la medición de los indicadores, se decidió utilizar una escala Likert de seis anclas a través de las cuales los respondientes debían indicar el grado de acuerdo/desacuerdo ( $1=$ Muy en desacuerdo a $6=$ Muy de acuerdo $)$ con cada afirmación contenida en el instrumento.

Evaluación de expertos. Para la valoración del banco inicial de ítems, se constituyó un grupo de discusión formado por seis expertos/as en género (dos por grado y dos del máster de secundaria) vinculados al Instituto Universitario de Investigación de Estudios de Género de la institución. Los expertos valoraron cualitativa y cuantitativamente el borrador inicial de 38 ítems. El análisis cualitativo consistió en someter a valoración la pertinencia, claridad y comprensión de cada uno de los ítems, así como las orientaciones incluidas en el cuestionario. Sus observaciones y/o comentarios contribuyeron a mejorar la redacción de algunos ítems confusos y, con ello, a incrementar la validez aparente de la primera versión de la ESFIG. Seguidamente, los evaluadores valoraron cuantitativamente su relevancia $(0=$ Nada relevante, $1=$ Algo relevante, $2=$ Relevante y $3=$ Muy relevante $)$ Tras esta valoración, fueron eliminados 10 ítems (aquellos cuya puntuación en la escala de relevancia fue inferior a dos). Aplicando la fórmula de Lawshe (1975) a los 28 ítems restantes se obtuvo un índice de validez de contenido del conjunto de la escala de .98. La versión preliminar de la ESFIG quedó comprendida por 28 ítems que, a su vez, fue revisada por dos expertas de la Unidad de Igualdad de la institución.

Pilotaje. La versión revisada de 28 ítems fue administrada en el curso 2016-2017 a un grupo de 175 estudiantes de los grados de educación infantil y primaria de los cursos $1^{\circ}$ a $4^{\circ}$. Tras los análisis de fiabilidad y validez efectuados, fueron eliminados seis ítems, aquellos que mostraban correlaciones con el constructo inferiores a .40. Por lo tanto, la versión inicial de la ESFIG que se utilizó en este estudio contenía 22 ítems.

\section{Procedimiento}

Administración. Tras haber obtenido el permiso de la institución y del profesorado, el cuestionario que contenía la ESFIG fue administrado en papel (segundo semestre del curso 2017-2018) a todos los grupos que componían las tres cohortes. Obtenido el consentimiento informado del alumnado de los respectivos grupos, se procedió a la administración de la escala durante la primera quincena del mes de abril. Las/os estudiantes cumplimentaron el cuestionario en tiempo de clase en presencia de una de las investigadoras. 
La participación fue voluntaria, anónima y confidencial y representaba al $63 \%$ del alumnado de grado matriculado en educación infantil, el 66\% del de educación primaria y el 54\% del máster en formación del profesorado de educación secundaria. Fueron entregados y cumplimentados un total de 610 cuestionarios, de los que nueve fueron eliminados por estar incompletos.

Análisis estadísticos. Los datos recogidos se analizaron en dos fases. En la primera, se examinó la validez de constructo de la escala a través de un análisis factorial exploratorio (AFE) seguido de un análisis factorial confirmatorio (AFC). Para determinar a priori sus dimensiones, se llevó a cabo el AFE utilizando el método de componentes principales con rotación varimax. Al objeto de retener factores significativos, se aplicaron tres criterios: (a) auto-valores mayores que uno, (b) selección de ítems con pesos factoriales por encima de .45, y (c) selección de factores con una carga factorial de al menos tres elementos (Costello y Osborne, 2005). Para dar sustento a la validez de constructo, se procedió a ejecutar el AFC. Debido a la naturaleza continua y distribución normal de los datos, se empleó el procedimiento de máxima verosimilitud, lo que nos permitiría verificar las dimensiones generadas en el AFE. Los índices de referencia tomados para determinar la viabilidad del modelo factorial generado del AFE y de la fundamentación teórica fueron: la ratio Chi cuadrado/grados de libertad $\left(\chi^{2} / g l\right)$, el Índice de Ajuste Comparativo (CFI), el Índice de Tucker-Lewis (TLI) y el Error Cuadrático Medio de Aproximación (RMSEA), teniendo en cuenta que, para tener un modelo de ajuste aceptable, el cociente $\chi^{2} / g l$ debe ser menor que 3 (Kline, 2016); CFI y TLI deben tener un valor por encima de .90 (el valor de estos índices varía de 0 a 1 , siendo más aceptables cuanto más se aproximan a 1), y RMSEA debe ser inferior a .08 (MacCallum et al., 1996). Un valor RMSEA menor de .05 es indicativo de un buen ajuste, mientras que valores por encima de .08 indican errores razonables de aproximación al modelo (Browne y Cudeck, 1993). La fiabilidad como consistencia interna fue explorada a través del coeficiente alpha de Cronbach. En la segunda fase, se calcularon estadísticos descriptivos para analizar las percepciones de los participantes acerca de la implementación de la PG en su formación, e inferenciales para identificar posibles diferencias de medias en las valoraciones efectuadas en función de la titulación y el género. La técnica utilizada para comparar las medias fue el análisis de la varianza entre grupos (ANOVA) de dos factores $(3 \times 2)$, tomando como primer factor la titulación (grado educación infantil $v s$. grado educación primaria $v s$. máster profesorado secundaria) y como segundo factor el género (mujer vs. varón). Todos los cálculos fueron realizados mediante el SPSS versión 24 y AMOS versión 23. 


\section{RESULTADOS}

\section{Validación}

Estructura factorial y fiabilidad. Como paso previo al AFE, se verificaron los supuestos de normalidad, simetría y curtosis para cada variable y se inspeccionó la matriz de correlaciones de los 22 ítems de la versión inicial de la ESFIG al objeto de eliminar aquellos de más baja asociación con el constructo. Como resultado del análisis, se eliminaron los ítems 1, 18, 21 y $22(r<.45)$. Tras comprobar la compatibilidad de los datos (pruebas de KMO y esfericidad de Bartlett), se realizó el AFE con los 18 ítems restantes utilizando el método de componentes principales. El gráfico de sedimentación mostró una solución de tres componentes que explicaba el $54.92 \%$ de la varianza (53.66\% muestra infantil, $56.85 \%$ primaria y $55.43 \%$ secundaria). No aparecieron ítems con pesos factoriales cruzados. Los ítems del Componente 1 se relacionaban con la sensibilidad institucional a la implementación de la política de igualdad de género; los del Componente 2, con la inclusión del género en el currículum; y los del Componente 3, con la conciencia de desigualdades asociadas al proceso de enseñanzaaprendizaje. Los índices de fiabilidad (alpha de Cronbach) obtenidos por subescala fueron $.89, .76$ y .76, respectivamente, índices que al ser mayores de .70 pueden considerarse satisfactorios (Nunnally y Bernstein 1994). La Tabla 1 contiene la solución factorial y descriptivos por titulación.

Análisis factorial confirmatorio. Basándonos en (a) la teoría del Doing Gender de West y Zimmerman (1987) y en los resultados del AFE, se especificó un modelo de tres factores y 18 ítems. Según recomiendan Marsh, et al. (2009), para confirmar las dimensiones, se realizó, primero, un AFC de primer orden (los 18 ítems cargados en un único factor, M1), y seguidamente, un segundo AFC utilizando la estructura de tres factores (M2).

Tabla 1

Matriz de componentes rotados del análisis de componentes principales y descriptivos por titulación

\begin{tabular}{|c|c|c|c|c|c|c|c|}
\hline & \multicolumn{3}{|c|}{ Factores } & \multirow{2}{*}{$\begin{array}{c}\begin{array}{c}\text { Total } \\
(N=601)\end{array} \\
\boldsymbol{M} / \boldsymbol{D T}\end{array}$} & \multirow{2}{*}{$\begin{array}{c}\underset{(n=196)}{\text { Infantil }} \\
\boldsymbol{M} / \boldsymbol{D T}\end{array}$} & \multirow{2}{*}{$\begin{array}{c}\underset{(n=202)}{\text { Primaria }} \\
\boldsymbol{M} / \mathbf{D T} \\
\end{array}$} & \multirow{2}{*}{$\begin{array}{c}\begin{array}{c}\text { ESO } \\
(n=203)\end{array} \\
\mathbf{M} / \mathbf{D T}\end{array}$} \\
\hline & I & II & III & & & & \\
\hline $\begin{array}{l}\text { Género en el } \\
\text { Currículum }\end{array}$ & & & & $5.08 / 0.88$ & $5.37 / 0.72$ & $\mathbf{5 . 0 0} / 0.89$ & $4.87 / 0.94$ \\
\hline $\begin{array}{l}\text { 7. Género integrado } \\
\text { en la docencia con } \\
\text { carácter obligatorio. }\end{array}$ & .818 & & & $\mathbf{5 . 2 1} / 1.09$ & $\mathbf{5 . 5 1} / 0.85$ & $\mathbf{5 . 1 0} / 1.14$ & $\mathbf{5 . 0 2} / 1.18$ \\
\hline
\end{tabular}




\begin{tabular}{|c|c|c|c|c|c|c|c|}
\hline & \multicolumn{3}{|c|}{ Factores } & \multirow{2}{*}{$\begin{array}{c}\begin{array}{c}\text { Total } \\
(N=601)\end{array} \\
\boldsymbol{M} / \boldsymbol{D T}\end{array}$} & \multirow{2}{*}{$\frac{\underset{(n=196)}{\text { Infantil }}}{\boldsymbol{M} / \boldsymbol{D T}}$} & \multirow{2}{*}{$\begin{array}{c}\text { Primaria } \\
(n=202) \\
\boldsymbol{M} / \boldsymbol{D T}\end{array}$} & \multirow{2}{*}{$\begin{array}{c}\begin{array}{c}\text { ESO } \\
(n=203)\end{array} \\
\mathbf{M} \boldsymbol{D} \boldsymbol{T}\end{array}$} \\
\hline & I & II & III & & & & \\
\hline $\begin{array}{l}\text { 13. Al menos una } \\
\text { asignatura sobre } \\
\text { igualdad en el plan. }\end{array}$ & .794 & & & $\mathbf{5 . 0 3 / 1 . 3 3}$ & $\mathbf{5 . 5 2} / 0.97$ & $5.00 / 1.13$ & 4.58/1.48 \\
\hline $\begin{array}{l}\text { 11. Mayor atención } \\
\text { a la diversidad de } \\
\text { identidades sexuales. }\end{array}$ & .788 & & & $4.84 / 1.16$ & $\mathbf{5 . 1 3} / 0.97$ & $4.74 / 1.17$ & $4.65 / 1.27$ \\
\hline $\begin{array}{l}\text { 12. Todas las } \\
\text { asignaturas impartidas } \\
\text { con PG. }\end{array}$ & .774 & & & $4.67 / 1.35$ & $\mathbf{5 . 1 1} / 1.13$ & $\mathbf{4 . 5 8} / 1.35$ & $4.33 / 1.45$ \\
\hline $\begin{array}{l}\text { 19. Perspectiva de } \\
\text { género esencial para } \\
\text { combatir el sexismo. }\end{array}$ & .759 & & & $\mathbf{5 . 2 1} / 1.00$ & $\mathbf{5 . 4 1 / 0 . 8 5}$ & $\mathbf{5 . 0 4 / 1 . 0 1}$ & $\mathbf{5 . 1 8} / 1.10$ \\
\hline $\begin{array}{l}\text { 20. Género igual de } \\
\text { importante que otras } \\
\text { diferencias. }\end{array}$ & .755 & & & $\mathbf{5 . 3 1 / 0 . 9 2}$ & $\mathbf{5 . 4 7 / 0 . 8 1}$ & $\mathbf{5 . 2 8} / 0.88$ & $\mathbf{5 . 1 8} / 1.03$ \\
\hline $\begin{array}{l}\text { 17. Formación } \\
\text { condición necesaria } \\
\text { para educar en IG. }\end{array}$ & .725 & & & $5.27 / 1.03$ & $\mathbf{5 . 4 2} / 0.93$ & $5.26 / 1.04$ & $\mathbf{5 . 1 4} / 1.11$ \\
\hline $\begin{array}{l}\text { Sensibilidad } \\
\text { Institucional }\end{array}$ & & & & $3.79 / 0.94$ & $3.84 / 0.88$ & $3.83 / 0.93$ & $\mathbf{3 . 7 1} / 0.99$ \\
\hline $\begin{array}{l}\text { 15. La facultad ha } \\
\text { adoptado un enfoque } \\
\text { proactivo hacia la IG. }\end{array}$ & & .771 & & $3.81 / 1.24$ & $\mathbf{3 . 9 5} / 1.15$ & $3.89 / 1.13$ & $\mathbf{3 . 6 1} / 1.40$ \\
\hline $\begin{array}{l}\text { 3. El género recibe } \\
\text { atención suficiente en } \\
\text { las asignaturas. }\end{array}$ & & .727 & & $3.39 / 1.35$ & $\mathbf{3 . 5 3} / 1.31$ & $3.37 / 1.33$ & $\mathbf{3 . 2 7} / 1.41$ \\
\hline $\begin{array}{l}\text { 16. La facultad aplica } \\
\text { la normativa sobre } \\
\text { igualdad. }\end{array}$ & & .690 & & $4.17 / 1.22$ & 4.25/1.09 & $4.16 / 1.18$ & 4.10/1.36 \\
\hline $\begin{array}{l}\text { 2. El plan desarrolla } \\
\text { la competencia para } \\
\text { educar en IG. }\end{array}$ & & .663 & & $3.87 / 1.52$ & $3.82 / 1.52$ & $3.94 / 1.54$ & $3.84 / 1.51$ \\
\hline $\begin{array}{l}\text { 14. El profesorado } \\
\text { está suficientemente } \\
\text { sensibilizado. }\end{array}$ & & .650 & & $3.73 / 1.25$ & $3.65 / 1.16$ & $3.79 / 1.23$ & $3.74 / 1.34$ \\
\hline $\begin{array}{l}\text { Conciencia } \\
\text { Desigualdades de } \\
\text { Género }\end{array}$ & & & & $\mathbf{2 . 2 1} / 0.96$ & $2.41 / 0.94$ & $2.15 / 0.93$ & $\mathbf{2 . 0 8} / 0.99$ \\
\hline
\end{tabular}




\begin{tabular}{|c|c|c|c|c|c|c|c|}
\hline & \multicolumn{3}{|c|}{ Factores } & \multirow{2}{*}{$\begin{array}{c}\begin{array}{c}\text { Total } \\
(N=601)\end{array} \\
\boldsymbol{M} / \boldsymbol{D T}\end{array}$} & \multirow{2}{*}{$\frac{\underset{(n=196)}{\text { Infantil }}}{\boldsymbol{M} / \boldsymbol{D T}}$} & \multirow{2}{*}{$\frac{\underset{(n=202)}{\text { Primaria }}}{M / D T}$} & \multirow{2}{*}{$\begin{array}{c}\begin{array}{c}\mathbf{E S O} \\
(n=203)\end{array} \\
\mathbf{M} / \boldsymbol{D T}\end{array}$} \\
\hline & $\mathbf{I}$ & II & III & & & & \\
\hline $\begin{array}{l}\text { 5. Los logros de } \\
\text { las estudiantes se } \\
\text { minimizan. }\end{array}$ & & & .778 & $\mathbf{2 . 2 3} / 1.31$ & 2.41/1.32 & $\mathbf{2 . 2 0} / 1.28$ & $2.07 / 1.33$ \\
\hline $\begin{array}{l}\text { 4. Los estudiantes } \\
\text { reciben más atención } \\
\text { que las estudiantes. }\end{array}$ & & & .723 & $1.81 / 1.16$ & $\mathbf{2 . 0 3} / 1.32$ & 1.71/1.04 & 1.68/1.09 \\
\hline $\begin{array}{l}\text { 10. Expectativas más } \\
\text { altas de los alumnos } \\
\text { que de las alumnas. }\end{array}$ & & & .719 & $\mathbf{2 . 0 3 / 1 . 1 0}$ & 2.17/1.12 & $1.93 / 1.04$ & $\mathbf{2 . 0 0 / 1 . 1 4}$ \\
\hline $\begin{array}{l}\text { 6. Los logros de } \\
\text { las estudiantes se } \\
\text { atribuyen al esfuerzo. }\end{array}$ & & & .717 & $\mathbf{2 . 8 0} / 1.47$ & $3.05 / 1.38$ & $2.77 / 1.52$ & $\mathbf{2 . 5 9 / 1 . 4 8}$ \\
\hline $\begin{array}{l}\text { 8. Mujeres docentes en } \\
\text { inferioridad respecto a } \\
\text { los docentes. }\end{array}$ & & & .514 & $\mathbf{2 . 8 1} / 1.45$ & $\mathbf{2 . 9 2 / 1 . 3 6}$ & $2.69 / 1.52$ & $\mathbf{2 . 8 2} / 1.47$ \\
\hline $\begin{array}{l}\text { 9. Poder ostentando } \\
\text { por los profesores. }\end{array}$ & & & .475 & $\mathbf{2 . 8 9} / 1.47$ & $\mathbf{2 . 8 2} / 1.41$ & $\mathbf{2 . 6 1} / 1.36$ & $3.23 / 1.57$ \\
\hline $\begin{array}{l}\text { KMO }=.854 \\
\text { Bartlett: } \\
\chi 2(153)=3961.50 \\
p<.000\end{array}$ & & & & & & & \\
\hline$\%$ varianza: $54.92 \%$ & 25.81 & 17.35 & 11.76 & & & & \\
\hline
\end{tabular}

Rango punt.: 1-6 (1-2 = Desacuerdo, 3-4 = Indeciso/a, 5-6 = Acuerdo $)$

El AFC del modelo $M_{1}$ (unifactorial) reveló índices de ajuste débil, según puede apreciarse en la Tabla $2:\left(\chi^{2} / 152\right)=962.50, \chi^{2} / g l=6.33$; RMSEA $=0.09, \mathrm{CFI}=.85 \mathrm{y}$ TLI $=.81$, por lo que explorando de nuevo los pesos factoriales de los elementos se decidió eliminar los ítems 8 y 9, dado que aparecían ligados a la conciencia de desigualdades de género en relación al profesorado, no al alumnado. Con los ítems 8 y 9 eliminados, se sometió a prueba el M2 (trifactorial) compuesto por los 16 ítems restantes que produjo índices de ajuste más satisfactorios: $\left(\chi^{2} / 117\right)=341.64, \chi^{2} / g l=2.92 ;$ RMSEA $=$ $0.07, \mathrm{CFI}=.92$ y TLI $=.91$. En base a estos resultados (Tabla 2$)$, se concluyó que la estructura de tres factores correlacionados $(r>.60)$ proporcionaba un mejor ajuste de los datos al modelo que la de un solo factor. 
Tabla 2

Estimaciones de bondad de ajuste del modelo

\begin{tabular}{lcccccc}
\hline & $\chi^{2}$ & gl & $\chi^{2} / \mathbf{g l}$ & CFI & TLI & RMSEA \\
\hline Nivel de ajuste recomendado & & & $<3$ & $>.90$ & $>.90$ & $<0.08$ \\
Modelo inicial $\left(\mathrm{M}_{1}\right)$ & 962.50 & 152 & 6.33 & .85 & .81 & 0.09 \\
Modelo revisado $\left(\mathrm{M}_{2}\right)$ & 341.64 & 117 & 2.92 & .92 & .91 & 0.07 \\
\hline
\end{tabular}

Nota. $\chi 2$ = Chi-cuadrado; $\mathrm{gl}$ = grados de libertad; CFI = Índice de Ajuste Comparativo; TLI = Índice de Tucker-Lewis; RMSEA = Error Cuadrático Medio de Aproximación

\section{Percepciones acerca de la implementación de la perspectiva de género}

Los estadísticos descriptivos organizados por factores se presentan en la Tabla 1. Teniendo en cuenta que el punto medio de la escala de medición es de 3.50, los resultados (total muestra) indican que los participantes valoraron (1) la sensibilidad del centro a la implementación de la política de igualdad de género de neutral o indiferente $(M=3.79, D T=0.94)$; (2) la inclusión del género en el plan de estudios altamente necesaria para su formación docente ( $M=5.08, D T=0.88)$; y (3) la conciencia de desigualdades de género baja $(M=2.21, D T=0.96)$, lo que indicaba que no percibían trato discriminatorio. El ítem de más alto acuerdo, como puede apreciarse en la Tabla 1, fue el 17 ( $M=5.27$, formación en género, condición necesaria para educar en igualdad) y el de más bajo consenso el $4(M=1.81$, los estudiantes reciben más atención del profesorado que las estudiantes).

La comparación de medias por titulación (Tabla 3) reveló que el alumnado de educación infantil percibía la inclusión del género en su formación más necesaria $(M=5.37)$ que el de primaria y secundaria $(M=5.00$ y 4.87 , respectivamente) $(p=.007)$; específicamente otorgaba una mayor importancia al carácter obligatorio $(p=.043)$ y transversal $(p=.001)$ que debía tener la incorporación de la PG en el currículum que el alumnado de primaria y secundaria. Los datos también revelaron que la conciencia de desigualdades de género era significativamente más baja en el alumnado de primaria $(M=2.15)$ y secundaria $(M=2.08)$ que en el de infantil $(M=2.41)$ $(p=.002)$. 
Tabla 3

Percepciones acerca de la aplicación de la perspectiva de género: comparación de medias según titulación y género (ANOVA $3 \times 2$ )

\begin{tabular}{|c|c|c|c|c|c|c|c|}
\hline & $\begin{array}{c}\text { Educación } \\
\text { Infantil } \\
\end{array}$ & $\begin{array}{c}\text { Educación } \\
\text { Primaria }\end{array}$ & $\begin{array}{l}\text { Educación } \\
\text { Secundaria }\end{array}$ & & & & \\
\hline & $M / D T$ & $M / D T$ & $M / D T$ & Efecto & $\boldsymbol{F}$ & $p$ & Eta \\
\hline \multirow{2}{*}{$\begin{array}{l}\text { Sensibilidad } \\
\text { institucional }\end{array}$} & $3.84 / 0.88$ & $\mathbf{3 . 8 3} / 0.93$ & $3.71 / 0.94$ & & & & \\
\hline & & & & $\mathrm{T}$ & 2.51 & .082 & .008 \\
\hline Mujer & 3.82/0.88 & $3.68 / 1.03$ & $3.62 / 1.03$ & G & 7.56 & $.006^{*}$ & .013 \\
\hline Varón & $4.16 / 0.94$ & $\mathbf{4 . 1 3} / 0.97$ & $\mathbf{3 . 8 2} / 0.95$ & $T \times G$ & 0.86 & .426 & .003 \\
\hline $\begin{array}{l}\text { Inclusión } \\
\text { género }\end{array}$ & $5.37 / 0.72$ & $\mathbf{5 . 0 0} / 0.89$ & 4.87/0.94 & $\mathrm{T}$ & 4.96 & $.007 *$ & .016 \\
\hline Mujer & $\mathbf{5 . 3 7 / 0 . 6 8}$ & $\mathbf{5 . 1 8} / 0.76$ & $\mathbf{5 . 1 1} / 0.77$ & G & 14.99 & $.000 *$ & .025 \\
\hline Varón & $5.22 / 1.25$ & $4.64 / 1.03$ & 4.57/1.04 & $T \times G$ & 0.99 & .371 & .003 \\
\hline $\begin{array}{l}\text { Conciencia } \\
\text { desigualdades }\end{array}$ & $\mathbf{2 . 4 1 / 0 . 9 4}$ & $2.15 / 0.93$ & $\mathbf{2 . 0 8} / 0.99$ & $\mathrm{~T}$ & 6.08 & $.002 *$ & .020 \\
\hline Mujer & $2.38 / 0.94$ & 2.19/0.87 & $\mathbf{2 . 0 5} / 0.92$ & G & 2.11 & .147 & .004 \\
\hline Varón & $\mathbf{2 . 9 5} / 0.71$ & $\mathbf{2 . 0 9} / 1.04$ & $\mathbf{2 . 1 1} / 1.06$ & $T \times G$ & 1.92 & .148 & .006 \\
\hline
\end{tabular}

Rango punt.: 1-6 (1-2 = Desacuerdo, 3-4 = Indeciso/a, 5-6 = Acuerdo); T = Titulación, G = Género, T x G = Interacción titulación x género; *Significativa $1 \%$

Las comparaciones por género (Tabla 3) indicaron que este influye en las percepciones de las/los estudiantes, de modo que el compromiso institucional con la aplicación de la política de igualdad de género fue peor valorado por las alumnas que por los alumnos $(M=3.71$ vs. 4.04, $p=.006)$. De hecho, aunque ambos consideraban que la PG no estaba recibiendo atención suficiente en las asignaturas $(M=3.23$ vs. 3.73), dicha atención fue significativamente peor valorada por las estudiantes que por los estudiantes $(p=.004)$, mientras que la actitud del centro hacia la igualdad fue considerada más proactiva por ellos que por ellas $(M=3.73$ $v s .4 .11, p=.015$ ). Las estudiantes fueron igualmente mucho más exigentes y críticas que sus compañeros a la hora de estimar la necesidad de incluir el enfoque de género en su formación $(M=5.22 v s .4 .81, p=.000)$, tamaño del efecto .025 bajo a moderado, pero significativo. Percibían en grado significativamente mayor que sus compañeros que la incorporación de la PG a la formación es esencial para combatir el sexismo $(p=.021)$ y que esta debía ser obligatoria $(p=.006)$ y transversal $(p=.003)$. El efecto de la 
interacción $T x G$ resultó estadísticamente no significativo, indicando que las diferencias encontradas en los tres colectivos atribuidas al género eran independientes de la titulación.

\section{DISCUSIÓN}

La contemplación de la igualdad de género como un objetivo común en Europa y en el mundo entero, y de la perspectiva de género como una estrategia para alcanzarla, hace necesarios estudios que exploren la incorporación del enfoque de género en los procesos formativos. La ausencia de instrumentos para medir el grado de implementación de la política de igualdad de género en el ámbito de la formación universitaria nos llevó, primero, a elaborar y validar un índice que permitiera explorar el estado de incorporación de la PG en el ámbito específico de la formación del profesorado; y segundo, una vez validado el instrumento, a utilizarlo para analizar y comparar las percepciones de los estudiantes de grado y máster en torno a (1) la aplicación institucional de la política de igualdad de género, (2) la integración de la perspectiva de género en el currículum y (3) la conciencia de las desigualdades de género asociadas a los procesos de enseñanza-aprendizaje.

Para el logro del primer objetivo, se diseñó la escala ESFIG, fundamentada en la teoría del Doing Gender (West y Zimmerman, 1987) y, tras obtener evidencia preliminar de sus propiedades (validez aceptable y buena fiabilidad), quedó compuesta en su versión final por 16 ítems y tres factores correlacionados distintos, pero complementarios. La confirmación de dichos factores hace posible el análisis de impacto de la política de igualdad de género a tres niveles (institucional, curricular e individual), por lo que el instrumento puede ser considerado útil para explorar el cumplimiento de la normativa de género en centros de formación del profesorado, identificar posibles carencias y necesidades, guiar intervenciones e investigar en docencia con PG en el ámbito de la formación inicial docente.

Con respecto al segundo objetivo, los resultados obtenidos de las valoraciones de los participantes revelan (a) percepciones de neutralidad e indiferencia institucional a la aplicación de la PG, (b) alto grado de acuerdo acerca de la necesidad de incorporar la PG a la docencia de forma obligatoria y transversal, y (c) baja conciencia de desigualdades de género en relación a los procesos instructivos. Los hallazgos revelan, asimismo, una mayor receptividad hacia la formación con PG en el alumnado de los grados de educación infantil y primaria que en el alumnado del máster en profesorado de educación secundaria, como también una percepción mucho más favorable a la formación en género en las estudiantes que en los 
estudiantes. Estos resultados son coherentes con la literatura y dan soporte a las conclusiones de otras investigaciones (e.g. Valdivieso, 2016) que sugieren que, a más alto nivel de etapa educativa, menor sensibilidad a la formación en género. Los hallazgos podrían estar apuntando a la fragilidad de las políticas de género y el escaso compromiso del centro y del profesorado como factores responsables; de hecho, autores diversos han denunciado la situación de dejadez en materia de formación para la igualdad de género en otras disciplinas distintas a la educación (e.g. Atchison, 2013; Larrondo y Rivero, 2019; Serra, et al., 2018; Verdonk, et al., 2009). Según Lombardo y Mergaert (2013), el esfuerzo por mantener el status quo puede haber llevado a las instituciones a hacer caso omiso de las reformas necesarias, cuyo resultado más visible es la indiferencia y/o resistencia a cualquier cambio curricular. Todo esto lleva a pensar que el centro de donde se extrajo la muestra del estudio ha podido no estar haciendo bien los deberes y, aunque semejante interpretación es especulativa, el estudio aporta elementos para la reflexión, el debate y la discusión de estas cuestiones en el seno del mismo.

Weiner (2000), en su revisión crítica de la incorporación del género en la preparación docente, analiza las semejanzas y diferencias de la situación en Europa y resume las formas más habituales de percibir e interpretar el mandato de la incorporación del género en la formación universitaria: (1) negación del problema; (2) género vinculado al feminismo; (3) amenaza a las relaciones de poder existentes, etc. A estas se añaden otras reacciones tomadas de la literatura estadounidense: no hay espacio para el género en el currículum; es un tema de baja prioridad; el género ya se enseña como parte de otros cursos sobre diversidad y multiculturalidad; es un tópico tabú, demasiado emocional y controvertido para ser tratado en el marco neutral y objetivo del aula universitaria (Hollingsworth, 1995). Así las cosas, no es de extrañar que reconociendo las dificultades que la implementación efectiva de la PG está teniendo dentro y fuera del ámbito educativo, el Secretario General de las Naciones Unidas, Ban Ki-moon, recomendara recientemente pasar rápidamente de los acuerdos y compromisos a la acción.

Otro hallazgo de interés en este estudio es la evidencia de invisibilidad de desigualdades de género con relación a los procesos instructivos que aportan los datos. El hecho de que el alumnado participante muestre una escasa conciencia de ellas ofrece apoyo a la idea de una percepción poco realista de las desigualdades que se originan en los procesos de enseñanzaaprendizaje y las relaciones de poder vinculadas al ámbito educativo. La división entre realidad e invisibilidad de desigualdades vinculadas al género ha sido puesta de relieve en estudios como el de Silva, et al. (2017) o Verge, et al. (2018). En el estudio de Verge, et al. (2018), por ejemplo, al ser preguntados un grupo de estudiantes de ciencias políticas acerca de sus perspectivas futuras una vez graduados, un alto porcentaje negaba la 
existencia de diferencias en oportunidad de acceso al trabajo, el éxito en la profesión o en la brecha salarial, datos que contribuyen a reafirmar que los/ las jóvenes no suelen ser conscientes de dichas desigualdades en la etapa formativa, a pesar de que ellas suelen ser más críticas que ellos. En educación, esa baja conciencia de desigualdades puede tener implicaciones serias para la práctica profesional futura, dado que sin la concienciación necesaria, las y los egresados difícilmente estarán en condiciones de pensar críticamente, identificar y/o reconocer acciones discriminatorias y desarrollar la competencia necesaria para educar en igualdad a las generaciones futuras. Por ello, se hace cada vez más necesaria una pedagogía sensible al género que ayude no solo a explorar e identificar desigualdades y desajustes, sino también a proporcionar apoyo continuado para desarrollar la competencia en género antes de abandonar la formación inicial universitaria.

Como limitaciones y proyecciones del estudio cabe mencionar las siguientes: Primero, la muestra, aunque de tamaño suficiente, fue extraída de una única institución, lo que compromete la generalización de los resultados más allá de la misma. Trabajar con muestras representativas extraídas de más universidades, facultades y disciplinas sería muy conveniente tanto para aportar evidencias en apoyo o no de los hallazgos y/o de la composición de la ESFIG, como para explorar su invarianza factorial, máxime teniendo en cuenta que algunos valores $F$ de la interacción T x G no superaron el valor de 1. Segundo, indagar en la relación de los factores de la ESFIG con variables institucionales (e.g. existencia de un ideario/ plan de género de centro), curriculares (e.g. presencia de asignaturas obligatorias de género en el programa de estudios) o del alumnado (e.g. haber cursado alguna asignatura sobre género) contribuiría a aportar evidencias de su validez convergente. Finalmente, aunque los resultados del estudio son teóricamente sólidos, convendría confrontar la visión del alumnado con la del profesorado y equipos directivos. Triangular las tres visiones combinando, además, técnicas cuantitativas y cualitativas, aportaría un conocimiento más comprensivo y profundo acerca de los motivos que pueden estar dificultando la incorporación de la PG en la formación inicial docente.

\section{CONCLUSIÓN}

La educación para la igualdad de género del alumnado de los grados y máster en formación del profesorado necesita ser atendida con urgencia, incorporando la visión de género a tres niveles (a) ideario y planes de centro, (b) currículo y programas de estudio, y (c) visión individual y conciencia de género. Los datos indican que la institución de donde se extrajo la muestra hasta ahora ha sido poco receptiva al cumplimiento de la política 
de igualdad de género, por lo que la PG como estrategia para formar a los/las futuros/as docentes en igualdad no está recibiendo la atención necesaria. Como consecuencia, las y los futuros docentes están perdiendo la oportunidad de adquirir competencias para forjarse una conciencia de género, de la desigualdad y de sus consecuencias, realista y no idealizada. La universidad no puede permanecer inamovible y distraída en otros asuntos por más tiempo. Su compromiso y esfuerzo continuado es vital y necesario para el bienestar, la convivencia y el desarrollo sostenible de la igualdad en las generaciones futuras.

\section{NOTAS}

Este estudio fue financiado por el Vicerrectorado de Calidad e Innovación Educativa (Instituto de Ciencias de la Educación) de la Universidad de Alicante, en el marco del programa Redes I3CE de investigación en docencia universitaria (Ref. 2018-2019/4413) y por el Instituto Universitario de Investigación de Estudios de Género, programa de Ayudas para el Desarrollo de Actividades Conducentes a la Realización de Tesis Doctorales (BOUA, 21/11/2019). 


\section{REFERENCIAS BIBLIOGRÁFICAS}

Anguita-Martínez, R. (2011). El reto de la formación del profesorado para la igualdad. Revista Interuniversitaria de Formación del Profesorado, 14(1), 4351.

Atchison, A. (2013). The practical process of gender mainstreaming in the political science curriculum. Politics and Gender 9(2), 228-235. https://doi. org/10.1017/S1743923X13000081

Ballarín-Domingo, P. (2017). ¿Se enseña coeducación en la universidad? Atlánticas, Revista Internacional de Estudios Feministas, 2(1), 7-31. https:// doi.org/10.17979/arief.2017.2.1.1865

Browne, M.W. \& Cudeck, R. (1993). Alternative ways of assessing model fit. En K. A. Bollen J. S. Long (Eds.), Testing structural equation models (pp. 136-162) SAGE.

Buquet, A.G. (2011). Transversalización de la perspectiva de género en la educación superior: problemas conceptuales y prácticos. Perfiles Educativos, XXXIII, 211-225.

Bustelo, M. (2003). Evaluation of gender mainstreaming: Ideas from a meta-evaluation study. Evaluation, 9(4), 383-403. https://doi. org/10.1177/135638900300900402

Costello, A.B. y Osborne, J.W. (2005). Best practices in exploratory factor analysis: Four recommendations for getting the most from your analysis. Practical Assessment, Research and Evaluation, 10, 1-9.

Garrigues, A. (2010). Proyecto integración de la perspectiva de género y las enseñanzas en materia de igualdad de mujeres y hombres y no discriminación en los planes de estudio de grado de la Universitat Jaume I. Resolución de la
Dirección General del Instituto de la Mujer de 13 de julio de 2009 (BOE, 22/07/2009).

González-Pérez, T. (2017). Políticas educativas igualitarias en España: la igualdad de género en los estudios de magisterio. Archivos Analiticos de Politicas Educativas, 26(2), 1-17. https://doi.org/10.14507/epaa.26.2764

Gudbjornsdottir, G.S., Thordardottir, T., \& Larusdottir, S.H. (2017, octubre). Gender equality issues in teacher education and in schools: A plea for a change in practice. [Comunicación] Conferencia Gender Training in Education, Lisboa, Portugal.

Hollingsworth, S. (1995). The 'problem' of gender in teacher education. MidWestern Educational Researcher, 8(2), 3-11.

Instituto de la Mujer - Red2Red Consultores. (2004). Informe final del estudio para conocer la situación actual de la educación para la igualdad en España. Red2Red Consultores.

Kline, R.B. (2016). Principles and practices of structural equation modeling ( $4^{\mathrm{a}}$ ed.). Guilford Press.

Kreitz-Sandberg, S. (2013). Gender inclusion and horizontal gender segregation: Stakeholders' strategies and dilemmas in Swedish teachers' education. Gender and Education, 25(4), 444-465. https://doi.org/10.1080 /09540253.2013.772566

Lahelma, E. \& Tainio, L. (2019). The long mission towards gender equality in teacher education: Reflections from a national project in Finland. Nordic Studies in Education, 39(1), 69-84. 
https://doi.org/10.18261/issn.18912019-01-06

Lam, S., Dodd, W., Whynot, J., \& Skinner, K. (2019). How is gender being addressed in the international development evaluation literature? A meta-evaluation. Research Evaluation, 28(2), 158-168. https://doi.org/10.1093/ reseval/rvy042

Larrondo, A. \& Rivero, D. (2019). A case study on the incorporation of gender-awareness into the university journalism curriculum in Spain. Gender and Education, 31(1), 1-14. https://doi.org/10.1080/09540253.2016 .1270420

Lawshe, C.H. (1975). A quantitative approach to content validity. Personnel Psychology, 28, 563-575. https:// doi.org/10.1111/j.17446570.1975. tb01393.x

Lombardo, E. \& Mergaert, L (2013). Gender mainstreaming and resistance to gender training: A framework for studying implementation. Nordic Journal of Feminist and Gender Research 21(4), 296-311. https://doi.or g/10.1080/08038740.2013.851115

MacCallum, R.C., Browne, M.W., \& Sugawara, H.M. (1996). Power analysis and determination of sample size for covariance structure modeling. Psychological Methods, 1(2), 130-149. https://doi.org/10.1037/1082989X.1.2.130

Marsh, H.W., Muthe'n, B., Asparouhov, T., Lüdtke, O., Robitzsch, A., Morin, A.J., \& Trautwein, U. (2009). Exploratory structural equation modeling, integrating CFA and EFA: Application to students' evaluations of university teaching. Structural Equation Modeling, 16, 439-476. https:// doi.org/10.1080/10705510903008220
Martínez, Y. y Díaz, Z. (2017). Evaluación sensible al género para la gestión de sistemas y servicios de salud. Revista Chilena de Salud Pública, 21(2), 160168. https://doi.org/10.5354/07195281.2017 .48916

Naciones Unidas (1979). Convención sobre la Eliminación de Todas las Formas de Discriminación contra la Mujer (CEDAW). https://bit. ly/2vuGqoQ

Naciones Unidas (2015). Transformar nuestro mundo: la Agenda 2030 para el Desarrollo Sostenible. https://bit. $\mathrm{ly} / 37 \mathrm{~g} 806 \mathrm{E}$

Nunnally, J.C. \& Bernstein, I.H. (1994). Psychometric theory. McGrawHill.

Palkki, J. \& Sauerland, W. (2019). Considering gender complexity in music teacher education. Journal of Music Teacher Education, 28(3), 72-84. https://doi. org/10.1177/1057083718814582

Pastor-Gosálbez, I., Acosta-Sarmiento, A., Torres-Coronas, T., y Calvo-Merino, M. (2020). Los planes de igualdad en las universidades españolas: situación actual y retos de futuro. Educación $X X 1,23(1), 147-172$. https://doi. org/10.5944/educXX1.23873

Romero, A. y Abril, P. (2008). Género y formación del profesorado en los estudios de educación infantil. Revista Electrónica Interuniversitaria de Formación del Profesorado, 11(3), 4351.

Serra, P., Soler, S., Prat, M., Vizcarra, M. T., Garay, B., \& Flintoff, A. (2018). The (in)visibility of gender knowledge in the Physical Activity and Sport Science degree in Spain. Sport, Education and Society, 23(4), 324-338. https://doi.org/ 10.1080/13573322.2016.1199016 
Silva, M. J., Ferreira, E., \& Ferreira, A. (2017). Gender awareness in teacher education: Towards an embodied and inclusive approach to elementary science education. Conexão Ciência, 12(2), 147-152.

UNESCO (2015a). Marco de Acción en Educación 2030. https://bit. ly/37noERI

UNESCO (2015b). A guide for gender equality in teacher education: Policy and practices. https://bit.ly/38nsNGG

United Nations Women Training Center (2015). Training for gender equality: Twenty years on. https://bit. ly/2uF7qCe

Valdivieso, S. (Coord.), Ayuste, A., Rodríguez-Menéndez, M.C. y VilaMerino, E. (2016). Educación y género en la formación docente en un enfoque de equidad y democracia. En I. Carrillo i Flores (Coord.), Democracia y educación en la formación docente (pp. 117140). Universidad de Vic-Universidad Central de Cataluña.

Verdonk, P., Benschop, Y., de Haes, H. Mans, L. y Lagro-Janssen, T. (2009). Should you turn this into a complete gender matter? Gender mainstreaming in medical education. Gender and Education, 21(6), 703-719. https://doi. org/10.1080/09540250902785905
Verge, T., Ferrer-Fons, M. y González, M.J. (2018). Resistance to mainstreaming gender into the higher education curriculum. European Journal of Women's Studies, 25(1), 86-101. https://doi. org/10.1177/1350506816688237

Vizcarra, M.T., Nuño, T., Lasarte, G., Aristizabal, P.. y Álvarez, A. (2015). La perspectiva de género en los títulos de grado en la Escuela Universitaria de Magisterio de Victoria-Gasteiz. Revista de Docencia Universitaria, 13(1), 297-318. https://doi.org/10.4995/ redu. 2015.6448

Weiner, G. (2000). A critical review of gender and teacher education in Europe. Pedagogy, Culture, and Society, 8(2), 233-247. https://doi. org/10.1080/14681360000200091

West, C. \& Zimmerman, D. (1987). Doing gender. Gender and Society, 1, 125-151. https://doi. org/10.1177/0891243287001002002

Zippel, K., Ferree, M.M., \& Zimmermann, K. (2016). Gender equality in German Universities: Vernacularising the battle for the best brains. Gender and Education, 28(7), 867-885. https://doi. org/10.1080/09540253.2015.1123229 


\section{APÉNDICE} (ESFIG)

Escala de Evaluación Sensible a la Formación en Igualdad de Género

\section{Parte I: Orientaciones}

Esta encuesta tiene como propósito explorar las percepciones acerca de la implementación de la perspectiva de género en la formación inicial de las/os futuros/as docentes. Utilizando una escala de seis puntos en donde 1 = Muy en desacuerdo y $6=$ Muy de acuerdo, indica, rodeando con un círculo, el número que mejor representa tu opinión con respecto a cada una de las afirmaciones contenidas en el cuestionario.

\begin{tabular}{|c|c|c|c|c|c|}
\hline $\mathbf{1}$ & $\mathbf{2}$ & $\mathbf{3}$ & $\mathbf{4}$ & $\mathbf{5}$ & $\mathbf{6}$ \\
\hline $\begin{array}{c}\text { Muy en } \\
\text { desacuerdo }\end{array}$ & Desacuerdo & $\begin{array}{c}\text { Algo en } \\
\text { desacuerdo }\end{array}$ & $\begin{array}{c}\text { Algo de } \\
\text { acuerdo }\end{array}$ & $\begin{array}{c}\text { De } \\
\text { acuerdo }\end{array}$ & $\begin{array}{c}\text { Muy de } \\
\text { acuerdo }\end{array}$ \\
\hline MD & D & AD & AA & A & MA \\
\hline
\end{tabular}

Tus respuestas contribuirán a conocer tu visión sobre el grado de receptividad de tu facultad y plan de estudios a la incorporación de la perspectiva de género a la formación de docentes. Si además de responder al cuestionario, estuvieras dispuesto/a a ser entrevistado/a, agradeceríamos nos lo comunicases a la siguiente dirección electrónica: cmc138@alu.ua.es

\begin{tabular}{|l|c|c|c|c|c|c|}
\hline Cuestionario & MD & D & AD & AA & A & MA \\
\hline $\begin{array}{l}\text { La facultad de educación ha adoptado } \\
\text { un enfoque proactivo hacia la } \\
\text { igualdad de género. }\end{array}$ & 1 & 2 & 3 & 4 & 5 & 6 \\
\hline $\begin{array}{l}\text { La facultad aplica la normativa } \\
\text { vigente en materia de igualdad. }\end{array}$ & 1 & 2 & 3 & 4 & 5 & 6 \\
\hline $\begin{array}{l}\text { La formación en género es una } \\
\text { condición necesaria para aprender a } \\
\text { educar en igualdad. }\end{array}$ & 1 & 2 & 3 & 4 & 5 & 6 \\
\hline $\begin{array}{l}\text { Incluir la perspectiva de género en la } \\
\text { formación docente es esencial para } \\
\text { combatir el sexismo. }\end{array}$ & 1 & 2 & 3 & 4 & 5 & 6 \\
\hline
\end{tabular}




\begin{tabular}{|l|l|l|l|l|l|l|}
\hline $\begin{array}{l}\text { Las cuestiones de género son igual de } \\
\text { importantes para mi formación que } \\
\text { las relativas otras diferencias. }\end{array}$ & 1 & 2 & 3 & 4 & 5 & 6 \\
\hline $\begin{array}{l}\text { La diversidad de identidades sexuales } \\
\text { debería recibir mayor atención en el } \\
\text { plan de estudios. }\end{array}$ & 1 & 2 & 3 & 4 & 5 & 6 \\
\hline $\begin{array}{l}\text { Mi plan de estudios incluye el } \\
\text { desarrollo de competencias para } \\
\text { educar en igualdad de género. }\end{array}$ & 1 & 2 & 3 & 4 & 5 & 6 \\
\hline $\begin{array}{l}\text { La perspectiva de género recibe } \\
\text { atención suficiente en las asignaturas. }\end{array}$ & 1 & 2 & 3 & 4 & 5 & 6 \\
\hline $\begin{array}{l}\text { El género debería estar integrado en la } \\
\text { docencia con carácter obligatorio. }\end{array}$ & 1 & 2 & 3 & 4 & 5 & 6 \\
\hline $\begin{array}{l}\text { Todas las asignaturas del plan de } \\
\text { estudios deberían ser impartidas con } \\
\text { PG. }\end{array}$ & 1 & 2 & 3 & 4 & 5 & 6 \\
\hline $\begin{array}{l}\text { Debería haber al menos una } \\
\text { asignatura obligatoria sobre igualdad } \\
\text { de género en el plan de estudios. }\end{array}$ & 1 & 2 & 3 & 4 & 5 & 6 \\
\hline $\begin{array}{l}\text { El profesorado está suficientemente } \\
\text { sensibilizado a las cuestiones de } \\
\text { género. }\end{array}$ & 1 & 2 & 3 & 4 & 5 & 6 \\
\hline $\begin{array}{l}\text { El profesorado suele tener } \\
\text { expectativas más elevadas y exigentes } \\
\text { de los alumnos que de las alumnas. }\end{array}$ & 1 & 2 & 3 & 4 & 5 & 6 \\
\hline $\begin{array}{l}\text { Los estudiantes reciben más atención } \\
\text { del profesorado que las estudiantes. }\end{array}$ & 1 & 2 & 3 & 4 & 5 & 6 \\
\hline $\begin{array}{l}\text { Los logros de las estudiantes se } \\
\text { minimizan con frecuencia. }\end{array}$ & 1 & 2 & 3 & 4 & 5 & 6 \\
\hline $\begin{array}{l}\text { Los logros de las estudiantes se } \\
\text { atribuyen más a su esfuerzo que a su } \\
\text { capacidad. }\end{array}$ & 1 & 2 & 3 & 4 & 5 & 6 \\
\hline
\end{tabular}




\section{Parte II: Datos demográficos}

1. Edad: años

2. Género: $\quad \square$ Mujer $\quad \square$ Varón $\quad \square$ Otra identidad

3. Nacionalidad:

4. Estudios:

$\square$ Grado de Maestro en Educación Infantil

$\square$ Grado de Maestro en Educación Primaria

Máster en Formación Profesorado de Educación Secundaria
5. Curso:
$\square 1^{\circ}$
$\square 2^{\circ}$ $3^{\circ}$ $4^{\circ}$

6. Especialidad/mención elegida:

7. Dedicación a los estudios:

$\square$ Total

$\square$ Parcial

8. Tu plan de estudios ¿requiere cursar de forma obligatoria alguna asignatura relacionada con los estudios de género?: $\quad \square$ Sí $\quad \square$ No

9. ¿Existe en tu plan de estudios alguna asignatura optativa sobre género/ igualdad de género?:

$\square$ Sí $\quad \square$ No

10. ¿Has cursado alguna asignatura sobre género/igualdad de género durante tu formación universitaria? $\square$ Sí $\square$ No

En caso afirmativo, ¿de cuántos créditos?

11. Utilizando una escala de diez puntos siendo 1 (Mínima) y 10 (Máxima), indica qué importancia otorgas a la formación para la igualdad de género:

¡Muchas gracias por tu colaboración! 


\section{PERFIL ACADÉMICO Y PROFESIONAL DE LOS AUTORES}

C. Miralles-Cardona. ORCID: http://orcid.org/0000-0002-2903-0970

Estudiante de doctorado en Estudios Interdisciplinares de Género de la Universidad de Alicante. Orientadora en salud mental, discapacidad e igualdad en el Northeast Metro Intermediate School District, White Bear Lake, MN. Email: cmc138@alu.ua.es

María C. Cardona-Moltó. ORCID: http://orcid.org/0000-0001-8132-1161

Catedrática del área MIDE de la Universidad de Alicante. IP del Grupo de Investigación Diversidad, Educación y Género (VIGROB-298), del Instituto Universitario de Investigación de Estudios de Género. Líneas de investigación: educación inclusiva e igualdad de género, diseño y validación de escalas, y formación del profesorado para la atención a la diversidad.

Email: cristina.cardona@ua.es

Esther Chiner. ORCID: http://orcid.org/0000-0002-1546-7071

Profesora Titular del área MIDE de la Universidad de Alicante. Miembro del grupo Diversidad, Educación y Género del Instituto Universitario de Investigación de Estudios de Género. Líneas de investigación: educación inclusiva e igualdad de género, inclusión digital de personas con discapacidad intelectual y formación del profesorado para la atención a la diversidad. Email: esther.chiner@ua.es

Fecha Recepción del Artículo: 15. Febrero. 2019

Fecha Modificación del Artículo: 27. Enero. 2020

Fecha Aceptación del Artículo: 30. Enero. 2020

Fecha Revisión para Publicación: 10. Febrero. 2020 
